\section{Scientific journal}

PHYSICAL AND MATHEMATICAL EDUCATION

Has been issued since 2013.

Науковий журнал

ФІЗИКО-МАТЕМАТИЧНА ОСВІТА

Видається 32013.
ISSN 2413-158X (online)

ISSN 2413-1571 (print)

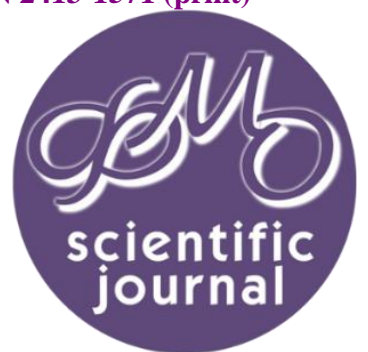

Тургунбаев Р.М. Принцип преемственности в обучении математическому анализу с помощью специально подобранных задач. Фізико-математична освіта. 2021. Випуск 2(28). С. 77-82.

Turgunbaev R. Principle of continuity in learning mathematical analysis using specially selected problems. Physical and Mathematical Education. 2021. Issue 2(28). P. 77-82.

DOI 10.31110/2413-1571-2021-028-2-013

Удк 378.016

P.М. Тургунбаев

Ташкентский государственный педагогический университет имени Низами, Узбекистан musamat1@yandex.ru

ORCID: 0000-0002-2264-6289

\title{
ПРИНЦИП ПРЕЕМСТВЕННОСТИ В ОБУЧЕНИИ МАТЕМАТИЧЕСКОМУ АНАЛИЗУ С ПОМОЩЬЮ СПЕЦИАЛЬНО ПОДОБРАННЫХ ЗАДАЧ
}

АННОТАЦИЯ

Формулировка проблемы. Рациональное применение принципа преемственности в обучении и достаточной для этого методики преподавания у преподавателя являются одним из условий успешного освоения первокурсниками математических дисциплин. С точки зрения тезаурусного подхода к обучению преемственность есть инъективное соответствие между лексиконом обучающегося и учебным тезаурусом дисциплины. Целью данной статьи является описать методику использования системы задач в рамках тезаурусного подхода на основе принципа преемственности в обучении математическому анализу в педагогическом вузе.

Материалы и методы. Материалами исследования являются педагогические и методические источники литературы, опыты зарубежных и отечественных педагогов. В ходе исследования были использованы методы: наблюдение и анализ для систематизации накопленной информации о целесообразности использования тезаурусного подхода в обеспечении преемственности в обучении математическому анализу, синтез лексикона студента и учебного тезауруса, моделирование системы задач для установления инъективного отображения между лексиконом студента и учебным тезаурусом, обобщение собственного педагогического опыта.

Результаты. Определены методы и средства обеспечивающие преемственности в обучении математическому анализу на основе тезаурусного подхода. На примере темы «Множества действительных чисел» построены системь специально подобранных задач.

Выводы. Решение студентом специально подобранных задач способствует пониманию студентом базовых понятий математического анализа и расширению его лексикона, освоению профессионального математического тезауруса. Предложенная система задач не претендует на полноту, она обладает свойствами гибкости, дополнения, модификации в зависимости от учебных задач и конкретной академической группы. Перспективы дальнейших исследований усматриваются в изучении вопроса эффрективного использования тезаурусного подхода в процессе обучения математическим дисииплинам.

КЛЮЧЕВЫЕ СЛОВА: лексикон студента, математический тезаурус, преемственности, множество действительных чисел, методика обучения, образовательная среда.

\section{ВВЕДЕНИЕ}

Постановка проблемы. От качества профессиональной подготовки учителей зависит успех реформ в сфере образования в стране, в частности работы по подъему школьного математического образования на новый уровень. Неотъемлемой частью профессиональной подготовки учителя математики является фундаментальная математическая подготовка.

Обеспечение фундаментальной математической подготовки учителей математики в педагогических вузах зависит от математической подготовки первокурсников, т.е. от знаний, умений, навыков, приобретённых в курсе общеобразовательной математики, уровня развития математического мышления. Однако наблюдения и исследования в этой области показывают, что уровень математической подготовки первокурсников недостаточен для того, чтобы они 
успешно освоили дисциплины «Математический анализ», «Геометрия», «Алгебра и теория чисел», которые изучаются на первом курсе. Наблюдаемые нами пробелы в математической подготовке первокурсников включают:

1) большинство студентов умеют решать стандартные задачи, но испытывают трудности с их обоснованием (проблемы алгоритмического мышления);

2) не знают и не понимают основных формулировок определений, теорем; не обладают навыками доказательств теорем, техникой вывода формул (проблема формального понимания содержания предмета);

3) навыки и компетенции самостоятельной работы практически отсутствуют.

В практике обучения в Высшей школе известны два способа выявления и устранения этих пробелов (Сманцер, 2013). Один из них - введение в учебный план дополнительного курса для повторения школьной математики в первом семестре, такой способ наблюдаются в некоторых педагогических вузах стран СНГ; второй - это рациональное применение принципа преемственности в обучении дисциплин и достаточной для этого методики преподавания у преподавателей.

В статье описана методика использования системы задач в рамках тезаурусного подхода на основе принципа преемственности в обучении математическому анализу по направлению образования «5110100-Математика и информатика». В предыдущих статьях (Тургунбаев,2019; Тургунбаев,2020) анализировались работы, проводимые в рамках тезаурусного подхода к обучению, важность преподавания предмета математического анализа с использованием метода тезауруса и создания предметного тезауруса в образовании.

\section{ТЕОРЕТИЧЕСКИЕ ОСНОВЫ ИССЛЕДОВАНИЯ}

Преемственность в математическом образовании - то многогранное понятие, которое по-разному трактовалось на разных этапах развития педагогики, психологии, методологии, как части прикладной философии. В статье (Жаров \& Тургунбаев, 2019) с точки зрения средовой педагогики и информационного подхода дано следующее определение.

Преемственность - инъективное соответствие, устанавливаемое специальным образом между личностной средой индивидуума, выражаемой лексиконом, и образовательной средой (внешней) посредством передачи сообщений (массивов данных) результатом, которого является активизация процессов интериоризации и экстериоризации индивидуума.

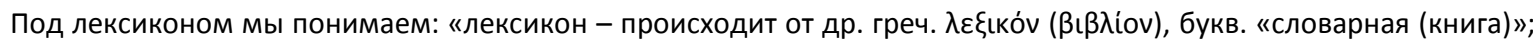
из $\lambda \dot{\varepsilon} \xi \iota \varsigma$ «слово». В русском языке слово «лексикон» - впервые употреблено у П. Берынды (1627 г.); заимствовано через нем. lexikon (XVII в.) или, подобно последнему, - книжным путем из лат. lexicon. Набор слов которыми владеет человек. Словарный запас. Различают два вида словарного запаса: активный и пассивный.

Активный словарный запас включает слова, которые человек использует в устной речи и письме.

Пассивный словарный запас включает в себя слова, которые человек знает при чтении или на слух, но не использует их сам в устной речи и письме. Пассивный словарный запас обычно больше активного в несколько раз» (Жаров \&Таратухина, 2016, с.37). Формирование активного профессионального словаря - результат профессиональной деятельности, тезаурус предмета.

Тезаурус (от греч. thesauros - сокровище, [От греч. Өпбаuрó - сокровище]): 1) словарь, в котором максимально полно представлены слова языка с примерами их употребления в тексте (в полном объеме осуществим лишь для мертвых языков); 2) словарь, в котором слова, относящиеся к каким-либо области знания, расположены по тематическому принципу и показаны семантические отношения (родовидовые, синонимические и др.) между лексическими единицами. В информационно-поисковых тезаурусах лексические единицы текста заменяются дескрипторами (Жаров \&Таратухина,2016, с. 64).

С точки зрения средовой педагогики тезаурус является частью образовательной среды и взаимодействует с личностной средой человека. Одной из проблем обучения человека является формирование его личного лексикона, в котором, придавая лексикону определенные свойства, преподаватели могут управлять процессом обучения, получения информации и построения профессионально ориентированного лексикона. Следует отметить, что тезаурус относительно статичен, а лексикон динамичен, поэтому лексикон отличается от тезауруса.

В исследованиях понятие студенческий лексикон также используется как личностный тезаурус (Гинецинский, 1992; Жаров, 2011). Таким образом, из приведенного выше определения следует, что для того, чтобы эффективно использовать преемственность в обучении, надо выяснить лексикон студента, который необходим для усвоения данной темы.

\section{МЕТОДЫ ИССЛЕДОВАНИЯ}

В ходе исследования были использованы ряд теоретических и эмпирических методов: наблюдение и анализ для систематизации накопленной информации о целесообразности использования тезаурусного подхода в обеспечении преемственности в обучении математическому анализу, синтез лексикона студента и учебного тезауруса, моделирование системы задач для установления инъективного отображения между лексиконом студента и учебным тезаурусом, обобщение собственного педагогического опыта.

\section{РЕЗУЛЬТАТЫ И ИХ ОБСУЖДЕНИЕ}

Опишем методику использования задач для установления преемственности между лексиконом студента и тезаурусом вышесказанной темы. Для этого мы составляем лексикон студента и предметный тезаурус, который должен быть усвоен студентом по теме «Множество действительных чисел» (Жаров \& Тургунбаев, 2019). Лексикон студента: натуральное число, целое число, рациональное число, числовая ось, начало координат, единичный отрезок, положительные числа, отрицательные числа, нуль, "лежит между", "лежит слева", "лежит справа", "соответствие", "ставить в соответствие", арифметические операции над рациональными числами, сравнение рациональных чисел, свойства числового неравенства, теорема Пифагора, построение с помощью линейки и циркуля (построение отрезка, который равен данному отрезку, построение перпендикуляра), теорема Фалеса. 
Математический тезаурус по теме «Множества действительных чисел» включает в себя: алгоритм определения точки (на прямой), соответствующей заданному рациональному числу, замкнутость множества рациональных чисел по отношению к арифметическим операциям; упорядоченность множества рациональных чисел; плотность множества рациональных чисел; отсутствие рационального числа, квадрат которого равно 2; рациональная точка, иррациональная точка; метод прямого доказательства, метод доказательства от противного; разбиение множества на части, сечение множества рациональных чисел, нижний класс, верхний класс, наименьший элемент, наибольший элемент, виды сечения, рациональное сечение, иррациональное сечение, сечение как число; иррациональное число, множество иррациональных чисел, действительное число, множество действительных чисел; сравнение действительных чисел, упорядоченность множества действительных чисел, свойство плотности множества действительных чисел, сечение множества действительных чисел, свойство непрерывности множества действительных чисел, десятичные аппроксимации действительных чисел, изображение действительного числа на числовой прямой; промежуток; сегмент; отрезок; интервал; полуинтервал; полусегмент; луч; ограниченное снизу множество; нижняя граница; точная нижняя граница; инфимум; минимальный элемент; ограниченное сверху множество; верхняя граница; точная верхняя граница; супремум; максимальный элемент; ограниченное множество; неограниченное множество; модуль; модуль действительного числа; неравенство треугольника; окрестность точки; геометрический смысл модуля; функция Дирихле.

Покажем систему, отобранных нами по указанной выше методике, задач, в которой в явной форме реализуется принцип преемственности в образовательном процессе. Суть как можно заметить состоит в том что мы устанавливаем необходимое нам соответствие между лексиконом студента-первокурсника и частью предметного математического тезауруса. Заметим, что некоторые из предложенных задач могут быть использованы при применении интерактивных методов на лекционных занятиях, а некоторые могут быть использованы на практических занятиях как дополнение к задачам данных в традиционных сборниках задач.

№1. Начертите числовую ось, дайте определение числовой оси, какие понятия входят в определение?

№2. Расскажите, как можно с помощью линейки и циркуля разделить данный отрезок на равные а) две части; б) три части.

№3. Постройте на числовой оси точки, соответствующие числам $1 ; 3 / 2 ; 4 / 3 ;-2,3$. Что можно сказать о расположения этих чисел относительно друг друга на числовой оси?

№4. Объясните значение словосочетаний «лежит между», «лежит слева», «лежит справа».

№5. Сформулируйте алгоритм построения точки на числовой оси, соответствующей заданному рациональному числу. Единственен ли такой алгоритм?

Задачи 1-3 даны для повторения (активизация неактивного лексикона) лексикона студентов. В задаче 2 деления отрезка на равные три части специально в школе не рассматривается, создаётся проблемная ситуация для студента. Решением 5-ой задачи основывается на знание алгоритма построения точки соответствующей заданному произвольному рациональному числу. Здесь студент должен абстрагироваться от конкретных рациональных чисел.

№6. Напишите общий вид рационального числа. Как определяется множество рациональных чисел?

№7. Докажите, что множество рациональных чисел замкнуто относительно арифметических операций. Инструкция. Для этого покажите, что сумма, разность, произведение, деление (где делитель отличен от нуля) любых двух рациональных чисел является рациональным числом. Запишите это свойство в символической форме.

Студент со школьной скамьи знаком с определением рационального числа, возможностью записать каждое рациональное число в виде несократимой дроби, выполнять арифметические операции над рациональными числами. Но эти факты не были объектом запоминания, специального изучения. Целью приведенных выше задач 6 и 7 является специальное внедрение в лексикон студента нового понятия множество рациональных чисел, его обозначения; свойства этого множества - замкнутость множества рациональных чисел относительно арифметических операции. Не трудно заметить, что уровень абстракции усвоения повышается.

В школе ученики изучают числовые неравенства, свойства числовых неравенств. Но с понятием упорядоченности множества и свойством упорядоченности студенты знакомятся в курсе математического анализа. Для введения этих понятии и свойств могут быть использованы следующие задачи.

№8. Когда одно рациональное число называется меньше (больше, равно) чем другое? Дайте геометрическое толкование этих понятии.

№9. Докажите утверждение: для любых двух рациональных чисел $r_{1}, r_{2}$ имеет место только одно из соотношений $r_{1}<r_{2}, r_{1}>r_{2}, r_{1}=r_{2}$.

№10. Докажите утверждение: пусть $r_{1}, r_{2}, r_{3} \in Q$. если $r_{1}<r_{2}, r_{2}<r_{3}$, то $r_{1}<r_{3}$. Дайте геометрическую интерпретацию этим утверждениям.

№11. Как можно определить отношения $\leq, \geq$ ? Докажите, что если $r_{1} \leq r_{2}$ и $r_{1} \geq r_{2}$, то $r_{1}=r_{2}$.

Для изучения свойства плотности множества рациональных чисел предлагаем следующие задачи.

№12. Всегда ли существует третье целое число между двумя неравными целыми числами? Обоснуйте ответ.

№13 Пусть 2/3 и 3/4 - рациональные числа. Приведите пример рационального числа больше чем 2/3, но меньше чем 3/4 (они «лежат между ними»). Сколько существует таких рациональных чисел? Можно ли указать способ нахождения рационального числа, которое находится между заданными рациональными числами?

№14. Обобщите вышеупомянутую задачу для любых двух неравных рациональных чисел. Это свойство называется свойством плотности множества рациональных чисел.

№15. Если неотрицательное рациональное число меньше любого положительного рационального числа, то оно равно нулю. Докажите.

Решая задачу №5, студенты констатируют факт, что каждому рациональному числу на числовой оси соответствует определенная точка. Следующий вопрос можно использовать, для мотивации необходимости пополнения множества рациональных чисел: 
№16. Выше мы отметили точку, соответствующую каждому рациональному числу. Это означает, что каждому рациональному числу соответствует ровно одна точка на числовой оси. Теперь, наоборот, соответствует ли каждой точке на числовой оси ровно одно рациональное число? Чтобы ответить на этот вопрос, мы делаем следующее: на числовой оси построим единичный отрезок, начало которого совпадает с началом координатной оси. Со второго конца отрезка построим перпендикуляр и с помощью циркуля на этом перпендикуляра отмерим единичный отрезок, начало которого совпадает с исходной точкой. Соединяем концы получившихся отрезков. В результате получим прямоугольный треугольник. С помощью циркуля на числовой оси с начала координат отмерим отрезок равный длине гипотенузы. Отметим второй конец этого отрезка через букву А. Квадрат длины этой гипотенузы равен 2 (теорема Пифагора). Соответствует ли рациональное число точке А? Напишите этот вопрос аналитически. (Существует ли несократимая дробь $\frac{p}{q}$ для которой $\left(\frac{p}{q}\right)^{2}=2$ ?)

Указание. Ответ: не существует. Предположите противное, то есть допустите, что «существует несократимая дробь $\frac{p}{q}$, для которой $\left(\frac{p}{q}\right)^{2}=2 »$ и получаете противоречие. Вспомогательная задача: квадрат четного числа четное число. Докажите это. Верно ли обратное?

№17. Из доказанного утверждения следует, что на числовой оси есть точки, не соответствующие рациональным числам. Точки, соответствующие рациональным числам, мы называем рациональными точками, остальные точки нерациональными (иррациональными) точками. Приведите примеры точек на числовой оси, которые не являются рациональными.

Понятие действительного числа в курсе математического анализа вводится на основе теории Дедекинда. Центральное понятие этой теории - сечение множества рациональных чисел. Для укрепления и развития лексикона, сформированной на лекции, на практических занятиях можно рекомендовать следующие задачи.

№18. Дайте определение понятию «сечение множества рациональных чисел». Как определяется сечение?

№19. Пусть А - множество отрицательных рациональных чисел, а В - множество положительных рациональных чисел. Образует ли множества А и В сечение? Обоснуйте ответ.

№20. Пусть $\mathrm{A}=Z$ - множество целых чисел, а В $=Q \backslash Z$ - остальные рациональные числа. Образует ли множества А и В сечение? Обоснуйте ответ.

№21. Пусть А - множество отрицательных рациональных чисел. Существует ли на этом множестве наибольший элемент (Существует ли наибольшее отрицательное рациональное число). Обоснуйте свой ответ.

№22. Пусть В - множество неотрицательных рациональных чисел. Есть ли в этом множестве наименьший элемент? Какой элемент наибольший?

№23. Пусть $A=\{r \in Q: r \leq 4\}$. Опишите множество А словесно. Каким должно быть множество В, чтобы А и В образовали сечение?

№24. Пусть $A=\left\{r \in Q: r \leq \frac{4}{3}\right\}, B=\left\{r \in Q: r>\frac{4}{3}\right\}$. Докажите, что эти множества образуют сечение. В этом случае говорят, что сечение (А, В) определяет число 4/3 (сечение первого рода).

Замечание. Сечение $(A, \mathrm{~B})$ также определяет число $\frac{4}{3}$, где $A=\left\{r \in Q: r<\frac{4}{3}\right\}, B=\left\{r \in Q: r \geq \frac{4}{3}\right\}$ (сечение второго рода).

№25. Постройте сечения, определяющие следующие числа: а) 2; б) -1,5. Нарисуйте эти сечения на числовой оси.

№26. Предположим, что множество А состоит из отрицательных рациональных чисел, нуля и положительных рациональных чисел квадрат которых меньше 2, а множество В состоит из положительных рациональных чисел квадрат которых больше 2. а) Докажите, что множества А и В образуют сечение. б) Докажите, что в нижнем классе А нет самого большого (максимального) элемента (числа). с) Докажите, что верхний класс В не имеет наименьшего (минимального) элемента (числа). Говорят, что это сечение определяет $\sqrt{2}$. Такое сечение называется сечением третьего рода (иррациональным).

№27. Постройте сечения, определяющие следующие числа: а) $\sqrt{3}$; б) $\sqrt[3]{2}$; в) $-\sqrt{2}$.

Далее рациональное сечение (для определенности - сечения первого рода) отождествляется рациональным числом, а сечение третьего рода - иррациональным числом. Т.е. сечение множества рациональных чисел является моделью действительного числа.

Для изучения основных свойств множества действительных чисел используются данная модель или язык «сечений». Для преемственного определения арифметических операций над действительными числами, свойства множества действительных чисел, так чтобы эти операции и свойства совпадали с исходными операциями над рациональными числами и свойствами множества рациональных чисел, студентам можно порекомендовать следующую систему задач, которую можно рассматривать как небольшую локальную теорию:

№28. Малая локальная теория (описание свойств множества рациональных чисел на языке «сечений»). Пусть, $r \in$ $Q$. Тогда $r=(A, B)$, где $A=\{x \in Q: x \leq r\}, B=\{x \in Q: r>x\}$, т.е. каждое рациональное число является сечением первого рода, и наоборот. В этом случае множество рациональных чисел состоит из множеств таких сечений.

28.1. Подумайте, как определить отношения «больше», «меньше», «равно» на языке «сечений». Нарисуйте на числовой оси.

28.2. Докажите свойства (плотность, упорядоченность) множества рациональных чисел на языке «сечений».

28.3. Как определить сложение (вычитание, умножение, деление) на языке «сечений»?

№29. Дайте определение иррациональному числу. Приведите примеры.

№30. Дайте определение действительного числа, множества действительных чисел. Как обозначается множество действительных чисел?

№31. Пусть $\alpha \in R ; \beta \in R$. Тогда по определению $\alpha=(A, B), \beta=(C, D)$, где $(A, B),(C, D)$ - сечения в множестве рациональных чисел. Дайте определение: а) $\alpha=\beta$; б) $\alpha<\beta$; в) $\alpha \leq \beta$; г) $\alpha>\beta$; д) $\alpha \geq \beta$. Проиллюстрируйте на числовой оси. Можно ли упростить определение в частном случае, когда одно из чисел рациональное? 
№32. Сформулируйте и докажите свойство плотности множества действительных чисел R.

№33. Сформулируйте и докажите свойство упорядоченности множества действительных чисел R.

№34. Объясните смысл теоремы Дедекинда, докажите теорему. Разделите доказательство теоремы на шаги, опишите его в виде блок-схемы. Как вы думаете, в чем заключается идея доказательства теоремы?

№35. Объясните, как определяются десятичные приближения действительного числа.

№36. Каждому действительному числу можно присвоить бесконечную десятичную дробь. Докажите, что любое конечное или периодическое бесконечное десятичное число представляет собой рациональное число.

№37. Какие из следующих бесконечных десятичных дробей являются рациональным числом: а) 2,13 (14); б) 2,76

(11); в) 0,4212121 ...; г) 0,1010010001 ...; д) 1,320320032 ...? Запишите рациональное число в виде обыкновенной дроби.

№38. Докажите, что следующая бесконечная десятичная дробь не является периодической: 0.1010010001 ....

№39. Объясните, как построить «иррациональную точку» на числовой оси.

Таким образом, для каждого действительного числа на числовой оси ставится одна точка, и наоборот. Такое соответствие называется взаимно однозначным (биекцией).

В курсе общеобразовательной математики учащиеся изучают понятие модуля, его геометрическую интерпретацию, методы решения уравнений и неравенств с модулями. Надо отметить, что свойства модуля в школе не доказываются. В курсе математического анализа изучаются понятие модуля действительного числа и его свойства. Для формирования понятия модуля и связанного с ним понятия, введение в лексикон студентов предметный тезаурус, связанный с модулем, можно осуществить с помощью следующих задач.

№40. Дайте определение модуля действительного числа, укажите его геометрическое значение. Можно ли назвать модуль действительного числа функцией? Укажите область определения и множество значений этой функции.

№41. Модуль рационального числа и его свойства изучались в школе. Можно ли обобщить эти свойства на множестве действительных чисел? Чем отличаются эти модули?

№42. Сформулируйте свойства модуля действительного числа, докажите их.

№43. Дайте определения основным числовым промежуткам (интервал, отрезок, полуинтервал, луч). Как они обозначаются? Нарисуйте их на числовой оси. Как вы думаете, что является их общим свойством (что их объединяет как промежутки)? Для каких промежутков всегда существует а) наибольший; б) наименьший элементы?

№44. Дайте определение окрестности точки, приведите примеры. Каково количество окрестности точки? Как она обозначается? Нарисуйте на числовой оси.

№45. а) Дайте определение ограниченному снизу (сверху) множеству. Дайте геометрическую интерпретацию. Приведите примеры. Какие из промежутков ограничены снизу, сверху? б) Дайте определение ограниченного множества, дайте геометрическую интерпретацию.

№46. Дайте определение нижней (верхней) границы. Каково количество нижних (верхних) границ?

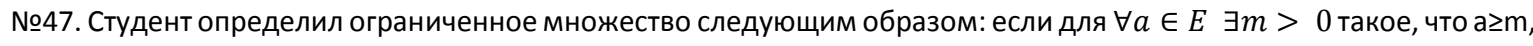
то E называется ограниченным снизу. Прав ли студент? Обоснуйте ответ. №48. Предположим, что а) $\mathrm{E}=[0 ; 2]$; б) $\mathrm{E}=(0 ; 2)$. Найдите множество верхних границ этих множеств.

№49. Пусть E - ограниченное множество сверху. Множество всех его верхних границ обозначим через В. А множество остальных действительных чисел через А. Обоснуйте, что А и В образует сечение множества действительных чисел. Докажите, что в верхнем классе существует наименьший элемент.

№50. Дайте определение точной верхней (нижней) границы. Как она обозначается?

№51. Докажите теорему: если множество Е ограничено снизу, то существует точная нижняя граница множества $\mathrm{E}$. №52. Объясните на примере, чем различаются понятия точной верхней границы и наибольшего элемента множества.

№53. Приведите пример неограниченного множества. Дайте определение неограниченного множества. №54. Множество натуральных чисел неограниченно. Докажите.

№55. Объясните на примерах разницу между «неограниченными» и «бесконечными» множествами.

\section{ВЫВОДЫ И ПЕРСПЕКТИВЫ ДАЛЬНЕЙШЕГО ИССЛЕДОВАНИЯ}

Некоторые из вышеперечисленных учебных вопросов кажутся простыми и очевидными, некоторые можно решить устно. Но, решая эти задачи, студенты активируют неактивную часть своего словарного запаса, связывая ее с новыми понятиями. То есть между лексиконом студента-первокурсника и предметным математическим тезаурусом устанавливается связь, происходит преобразование предметного тезауруса в личностный тезаурус студента, в результате этого достигается методическая цель - понимание базовых понятий математического анализа студента и расширяется его лексикон. Достигается и другая цель построенной нами методики происходит освоение части профессионального математического тезауруса как системы научного знания. Предложенная система задач не претендует на полноту, она обладает свойствами, гибкости, дополнения, модификации в зависимости от учебных задач и конкретной академической группы. Было бы целесообразно включить в данную систему задачи, в которых рассматривалась: замкнутость/не замкнутость множества иррациональных чисел относительно арифметических операций; задачи, связанные с арифметическими операциями между рациональными и иррациональными числами. Задачи на доказательство утверждений с кванторами существования и общности, на построения отрицания таких утверждений с использованием универсальной знаковой системы мы считаем является естественным образовательным фоном математики современной общеобразовательной школы. В экспериментальной части наших исследований мы, в некоторых сильных группах, одновременно показываем стили мышления теоретической и прикладной, непрерывной и дискретной математики. Это очень важный «побочный» эффект изучения курса математического анализа, где воспитание инфинитезимального мышления сопрягается с дискретным математическим мышлением. 


\section{Список использованных источников}

1. Гинецинский В.И. Основы теоретической педагогики. СПб.: Изд-во С.-Петерб. ун-та, 1992. 152 с.

2. Жаров В.К., Таратухина Ю.В. Феноменология крос-культурного образования. М.: «Янус-К». 2016. 134 с.

3. Жаров В.К., Тургунбаев Р.М. Проблема преемственности в методике преподавания математики и её интерпретации в современных образовательных школах. Вестник РГГУ. Серия Информатика. Информационная безопасность. Математика. 2019. №2. С. 52-74.

4. Информационно-педагогическая среда современного вуза. Коллективная монография. Под. Ред. Проф. Жарова В.К. М.: «Янус-К», 2011. 268 с.

5. Кожокарь О.П. Учебный тезаурус как средство развития понятийного мышления в процессе подготовки будущих учителей. Вестник Сургутского педагогического университета. 2012. №2. С. 115-119.

6. Сманцер А. П. Теория и практика реализации преемственности в обучении школьников и студентов. Минск : БГУ, 2013. $271 \mathrm{c}$.

7. Тургунбаев Р.М. Методика формирования тезауруса по дисциплине Математический анализ и её значение. Научный вестник ТГПУ. 2019. №2(19). С. 24-27.

8. Тургунбаев Р.М. Использование метода тезаурусов в обучении студентов математическому анализу. Научный вестник НамГУ. 2020. №7. С. 472-478.

\section{References}

1. Ginecinskij V.I. (1992). Osnovy teoreticheskoj pedagogiki. [Foundations of theoretical pedagogy] SPb.: Izd-vo S.-Peterb. unta. [in Russian]

2. Zharov V.K. \& Taratuhina Ju.V. (2016) Fenomenologija kros-kul'turnogo obrazovanija. [Phenomenology of cross-cultural education] M.: «Janus-K». [in Russian]

3. Zharov V.K. \& Turgunbaev R.M. (2019). Problema preemstvennosti v metodike prepodavanija matematiki i ejo interpretacii v sovremennyh obrazovatel'nyh shkolah [The issue of continuity in the methodology of teaching mathematics and its interpretation in modern education practices] Vestnik RGGU. Serija Informatika. Informacionnaja bezopasnost'. Matematika RSUH/RGGU BULLETIN. "Information Science. Information Security. Mathematics" Series Academic Journal, 2, 52-74. [in Russian]

4. Zharov V.I. (ed.). (2011). Informacionno-pedagogicheskaja sreda sovremennogo vuza. [Information and pedagogical environment of a modern university]. Kollektivnaja monografija - Collective monograph. M.: «Janus-K». [in Russian]

5. Kozhokar' O.P. (2012). Uchebnyj tezaurus kak sredstvo razvitija ponjatijnogo myshlenija $v$ processe podgotovki budushhih uchitelej. [Educational thesaurus as a means of developing conceptual thinking in the process of preparing future teachers]. Vestnik Surgutskogo pedagogicheskogo universiteta - Bulletin of the Surgut Pedagogical University, 2, 115-119. [in Russian]

6. Smancer A. P. (2013). Teorija i praktika realizacii preemstvennosti v obuchenii shkol'nikov i studentov [Theory and practice of implementing continuity in teaching schoolchildren and students]. Minsk : BGU. [in Russian]

7. Turgunbaev R.M. (2019) Metodika formirovanija tezaurusa po discipline Matematicheskij analiz i ejo znachenie [Methodology for the formation of a thesaurus for the discipline Mathematical analysis and its meaning]. Nauchnyj vestnik TGPU - Scientific Bulletin of TSPU, 2(19), 24-27. [in Uzbek]

8. Turgunbaev R.M. (2020). Ispol'zovanie metoda tezaurusov v obuchenii studentov matematicheskomu analizu [Using the thesaurus method in teaching students mathematical analysis]. Nauchnyj vestnik NamGU - Scientific Bulletin of NamSU, 7 472-478 [in Uzbek].

\section{PRINCIPLE OF CONTINUITY IN LEARNING MATHEMATICAL ANALYSIS USING SPECIALLY SELECTED PROBLEMS Riskeldi Musamatovich Turgunbaev}

Abstract.

Tashkent State Pedagogical University named after Nizami, Uzbekistan

Formulation of the problem. The rational application of the principle of continuity in teaching and a teacher's teaching methodology sufficient for this is one of the conditions for the successful mastering of mathematical disciplines by freshmen. From the point of view of the thesaurus approach to teaching, continuity is an injective correspondence between the student's vocabulary and the educational thesaurus of the discipline. The purpose of this article is to describe the methodology for using the system of tasks within the framework of the thesaurus approach based on the principle of continuity in teaching mathematical analysis at a pedagogical university.

Materials and methods. The research materials are pedagogical and methodological literature, the experiences of foreign and domestic teachers. In the course of the study, the following methods were used: observation and analysis to systematize the accumulated information about the expediency of using the thesaurus approach in ensuring continuity in teaching mathematical analysis, synthesis of the student's vocabulary and the educational thesaurus, modeling the system of tasks to establish an injective mapping between the student's vocabulary and the educational thesaurus, generalizing one's own teaching experience.

Results. Methods and means are identified that ensure continuity in teaching mathematical analysis based on the thesaurus approach. On the example of the topic "Sets of real numbers", systems of specially selected problems are constructed.

Conclusions. In the course of the study, it is shown that the solution by the student of specially selected problems contributes to the student's understanding of the basic concepts of mathematical analysis and the expansion of its lexicon, the development of a professional mathematical thesaurus. The proposed system of tasks does not claim to be complete, it has properties, flexibility, additions, modifications, depending on the educational tasks and a specific academic group. Prospects for further research are seen in the study of the issue of the effective use of the thesaurus approach in the process of teaching mathematical disciplines.

Key words: student lexicon, mathematical thesaurus, continuity, set of real numbers, teaching methods, educational environment.

\section{$(\mathrm{Cc}) \mathrm{BY}-\mathrm{NC}-\mathrm{SA}$}

This work is licensed under Creative Commons Attribution-NonCommercial-ShareAlike 4.0 International License. 